\title{
Effect of shape and size of sampling window on the determination of average length, intensity and density of trace discontinuity
}

\author{
A. Kamali \& K. Shahriar \\ Department of Mining and Metallurgical Engineering, Amirkabir University of Technology, Tehran, Iran \\ M. Sharifzadeh \\ Department of Mining Engineering and Metallurgy Engineering, WASM, Curtin University, Australia
}

A. Aalianvari

Department of Mining Engineering, Faculty of engineering, University of Kashan, Kashan, Iran

\author{
A. Esmaeilzadeh \\ Department of Mining and Metallurgical Engineering, Urmia University of Technology, Urmia, Iran
}

\begin{abstract}
The fractures geometrical characteristics can be calculated by various sampling methods in 1 dimension (1D) \& 2 dimensions (2D). The Discrete Fracture Network (DFN) simulation results show some of the equations are suitable for calculation of mean trace length and the difference between actual value and their values is less than $15 \%$. Apparent density is dependent on scale, but the Mauldon's estimators are independent of scale despite the variation in fracture length. For all joint sets, the difference of apparent areal intensity by samplings windows and circular estimator is less than $5 \%$ proportionate to actual value. A reduction of Fisher constant doesn't have much effect on mean trace length of the fractures with length less than $1.5 \mathrm{~m}$, but it results in a $21 \%$ difference for fractures with length longer than $1.5 \mathrm{~m}$. Variation of this parameter affects the density, too and the difference can be 5\% to 10\% depending on fracture length. But, variation in Fisher constant doesn't have any effect on areal intensity for fractures with length less than $2 \mathrm{~m}$.
\end{abstract}

\section{INTRODUCTION}

Characterization of fractures has an important role in describing the rock mass. Seeing that the rock mass structure cannot be investigated directly in three dimensions, thus fractures' characterization is normally described on limited area (area sampling) or scanline (Zhang et al. 2010). Trace length, intensity and density are important and commonly-used characterizations. These characteristics for estimating the elastic properties of the rock, porosity of fractures and rock mass description for determining its hydraulic and mechanical behavior with usage in hydrogeology, oil recovery, evaluation of hydrocarbon flow, storage in fractured reservoir and rock engineering, rout length and connectivity of fractures in fluid flow are beneficial and important (Mauldon et al. 1999 a, b, Rohrbaugh 2002). The determination of the three above characterizations is necessary for constructing and verifying of the Discrete Fracture Network (DFN) (Kulatilake 2003, Reeves et al. 2013, Lei 2014). The estimation of these characteristics in field encounters problems as censoring and different types of biases such as length and orientation. Above characterizations are estimated by scanline, window or areal and circular sampling methods. Scanline sampling is a quick method for recording the fracture characterization, but it has size, truncation and censoring biases (Zhang et al. 2010, Zeeb et al. 2013).

There are various methods as regards the importance and the main role of fracture trace length in the investigation of rock mass behavior and necessity of fracture size calculation. These methods are developed by Mauldon, Zhang \& Einstein, Anderson \& Dverstrop, Einstein \& Baecher, Laslett and Pahl (Zhang et al. 2010, Ferrero et al. 2011). These methods use rectangle and circular windows for determining the fracture trace length. The developed equations by Mauldon, apparent density and apparent intensity equations proportionate to rectangle, circular windows and scanline are used to calculate of density and intensity, too (Zeeb et al. 2013, Mauldon et al. 2001). There has been no study the effect of Fisher constant (dispersion of fractures pole around the mean pole) and the shape of window sampling considering four joint sets on the above three characterizations so far (to authors' knowledge). 
Table 1. The used equations for calculation of mean trace length.

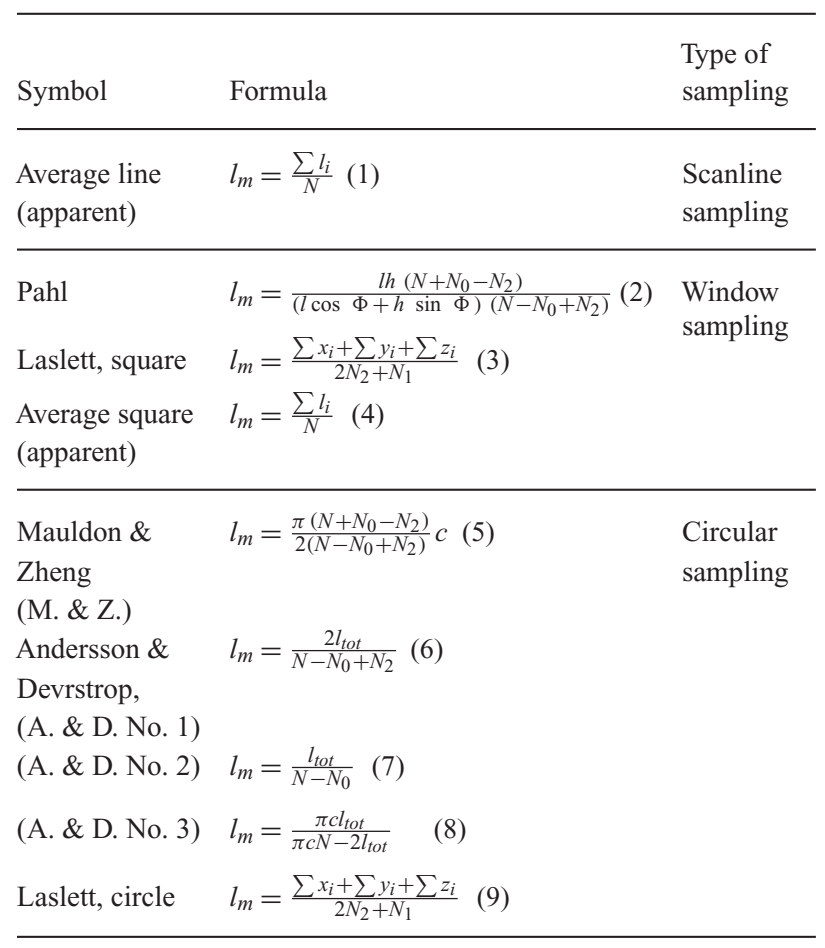

\section{BACKGROUND}

\subsection{Definition of above characteristics and equations}

The discontinuity trace length is formed by a planar fracture intersection with a surface such as rock face or bedding plan (Brown 1981). This is considered as one the most important rock mass characterizations, but it is one the most difficult characterizations for quantitating.

Intensity has three types including: linear $\left(\mathrm{P}_{10}\right)$, areal $\left(\mathrm{P}_{21}\right)$, and volumetric $\left(\mathrm{P}_{32}\right)$. The dimension of all types is $\mathrm{L}^{-1}$. Areal intensity or trace intensity is the length of fracture trace per unit sampling area.

Density has three types including: linear $\left(\mathrm{P}_{10}\right)$, areal (trace density) $\left(\mathrm{P}_{20}\right)$ and volumetric $\left(\mathrm{P}_{30}\right)$. They are number of trace centers per unit length of scanline, per unit sampling area and per unit rock volume, respectively. The dimension of these types is $\mathrm{L}^{-1}, \mathrm{~L}^{-2}$ and $\mathrm{L}^{-3}$, respectively. Estimation of these characterizations is effected by length and censoring biases (Mauldon et al. 1999a, Mauldon et al. 2001, Dershowitz et al. 1992).

As regards the sampling methods, various equations and estimators for calculation of mean trace length, intensity and density exist. These equations are shown in Tables 1, 2 and 3.

\subsection{Study rock mass characteristics by developed 3DDFNE code (3DAVOSHT)}

A three dimension discrete fracture network code (3DAVOSHT) in Matlab script is developed to calculate the parameters of equations 1 to 18 , investigate the type of shape and size of window sampling effects and
Table 2. The used equations for calculation of areal density.

\begin{tabular}{lll}
\hline Symbol & Formula & $\begin{array}{l}\text { Type of } \\
\text { sampling }\end{array}$ \\
\hline $\begin{array}{l}\text { Apparent } \\
\text { square Mauldon }\end{array}$ & $\frac{N_{1}+N_{2}+N_{0}}{l h}(10)$ & Window sampling \\
\hline$P_{20}=\frac{2 N_{2}+N_{1}}{2 l h}(11)$ & \\
\hline $\begin{array}{l}\text { Apparent } \\
\text { circle }\end{array}$ & $\frac{N_{1}+N_{2}+N_{0}}{\pi c^{2}}(12)$ & Circular sampling \\
$\begin{array}{l}\text { Mauldon } \\
\text { Method }\end{array}$ & $\frac{N-N_{0}+N_{2}}{2 \pi c^{2}}(13)$ & \\
\hline
\end{tabular}

Table 3. The used equations for calculation of linear and trace density.

\begin{tabular}{lll}
\hline Symbol & Formula & $\begin{array}{l}\text { Type of } \\
\text { sampling }\end{array}$ \\
\hline $\begin{array}{l}\mathrm{P}_{10} \\
\text { Line } \\
\text { method }\end{array}$ & $\begin{array}{l}P_{10}=\frac{1}{S}(15) \\
\text { Scanline sampling }\end{array}$ & \\
\hline $\begin{array}{l}\text { Apparent } \\
\text { square }\end{array}$ & $\frac{\sum x_{i}+\sum y_{i}+\sum z_{i}}{l h}(16)$ & Window sampling \\
\hline $\begin{array}{l}\text { Apparent } \\
\text { circle }\end{array}$ & $\frac{l_{t o t}}{\pi c^{2}}(17)$ & Circular sampling \\
$\begin{array}{l}\text { Mauldon } \\
\text { Method }\end{array}$ & $\frac{N+N_{0}-N_{2}}{4 c}(18)$ & \\
\hline
\end{tabular}

Where: S:fracture spacing, N:total number of fractures, $\mathrm{N}_{0}$ :number of fractures with two censored endpoints, $\mathrm{N}_{2}$ :number of fractures with two visible endpoints, $\mathrm{N}_{1}$ :number of fractures with one visible endpoint, L:scanline length, $\mathrm{x}_{\mathrm{i}}$ :length of fracture with two visible endpoints, $y_{i}$ :length of fracture with one visible endpoint, $z_{i}$ :length of fracture with two censored endpoints, $1_{\text {tot }}$ :total length of visible fractures in sampling window, c:radius of sampling window, $1_{\mathrm{i}}$ :fracture length, $1_{\mathrm{m}}$ :mean trace length, $1 \&$ h:wide and width of window sampling, $\phi$ :angle between vertical side of window sampling and trace length, $\mathrm{P}_{10}$ :linear intensity, $\mathrm{P}_{20}$ :areal density

investigate the three surveying methods on mean trace length, intensity and density. At the base of Baecher disk model, the center of fractures is generated by Poisson's process (Baecher et al. 1977) and uniform distribution is used for the simulation of fractures location (Zheng et al. 2015). This code can models four joint sets with circular and elliptical shape in 3D. The geometric parameters of the joints can be defined as non-deterministic. For generating the discontinuity in 3D, eight input parameters including: dip and dip direction (with fisher distribution), mean and variance of trace length (with Gamma, Lognormal, Exponential and Weibull distribution functions), Fisher constant, $\rho$ angle (angle between scanline and joint's mean pole), frequency and joint diameter are necessary. The fisher constant shows depression of fractures pole around mean pole. 
Table 4. The dimensions of windows sampling.

\begin{tabular}{lll}
\hline $\begin{array}{l}\text { Areal } \\
\text { sampling } \\
\text { number }\end{array}$ & $\begin{array}{l}\text { Circular } \\
\text { sampling } \\
\text { radius }(\mathrm{m})\end{array}$ & $\begin{array}{l}\text { Window } \\
\text { sampling dimensions } \\
(\mathrm{m} * \mathrm{~m})\end{array}$ \\
\hline 1 & 1 & $1.8 * 1.8$ \\
2 & 1.5 & $2.7 * 2.7$ \\
3 & 2 & $3.5 * 3.5$ \\
4 & 2.5 & $4.4 * 4.4$ \\
5 & 3 & $5.3 * 5.3$ \\
6 & 3.5 & $6.2 * 6.2$ \\
7 & 4 & $7 * 7$ \\
\hline
\end{tabular}

After generating of the 3D discrete fracture network, the 2D sections can be drawn in any direction and even in an oblique direction at each location. So, regarding the $2 \mathrm{D}$ sections and by the three sampling methods, the above geometric characteristics will separately be calculated based on the above equations for all four joint sets.

\subsection{Fracture simulation}

There have been considered four synthetic joint sets with different and non-deterministic length and orientation to investigate on the variation of the above characteristics proportionate to type and size of the sampling windows and getting closer to the actual conditions.

Fisher distribution for definition of orientation is used to investigate more accurate. The results of simulations $1 \& 2$ and simulation 3 have been taken into consideration to study the size, shape of sampling window and fisher-constant effect, respectively. Gamma and circle in simulations have been considered the diameter distribution and shape of fractures, respectively. Fractures are generated in a block with dimensions $10 * 10 * 10 \mathrm{~m}^{3}$. Each simulation has 5 realizations and that their average is considered in calculations. In simulation nos. 2 and 3, the center of fractures is the same and only the fisher constant has been increased from 8 to 100 to investigate of Fisher constant. The number of generated fractures in simulations $1 \& 2$ are different; on a way that in 5 realizations, on average the number of fractures in the simulation 2 proportionate to the simulation 1 is increased about $27 \%$. Given that there are four joint sets in each simulation exist, each simulation has only five fracture network realizations.

To record the necessary information about the generated fractures, seven circular and square sampling windows with equal centers and seven scanline with $10 \mathrm{~m}$ length proportionate to window sampling are used in proper location. The specifications of sampling windows are shown in Table 4 . The horizontal axis of all graphs in next sections is proportionate to introduced values in Table 4 .

The circular and square windows have same area. The dimensions of the window are selected based on the actual conditions in tunnels and slopes.

2D section together with scanline and sampling windows with radius of $2.5 \mathrm{~m}$ and $4.4 * 4.4 \mathrm{~m}$

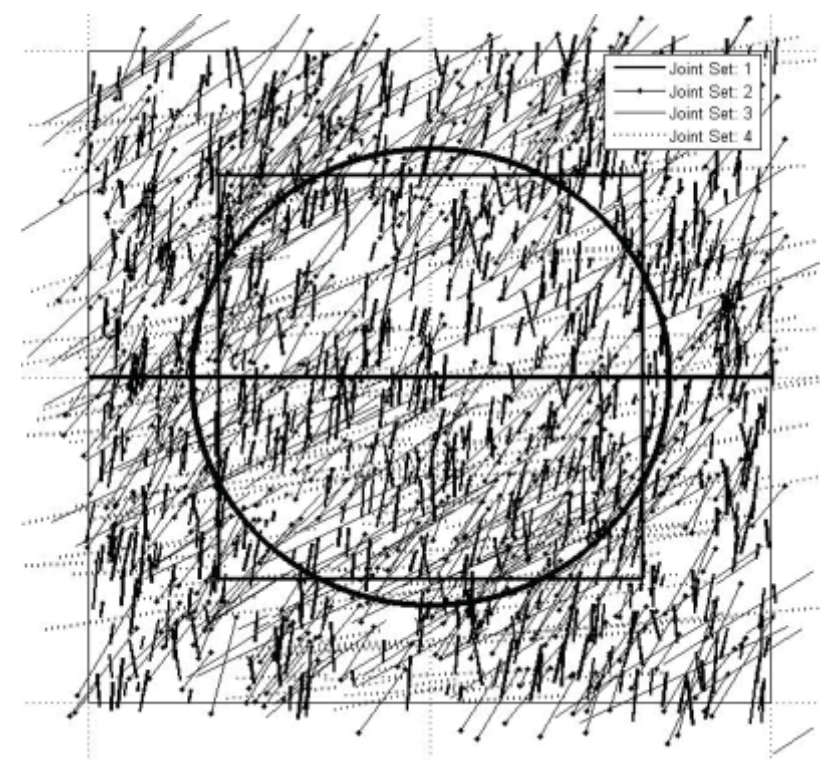

Figure 1. 3D model of four joint sets network and 2D section with scanline and windows sampling (circle and square), Fisher constant is equal 100.

dimension (type 4 of sampling window) are shown in Figure 1.

In simulations, whole sampling windows and scanline are located in $\mathrm{x}=5 \mathrm{~m}$ of $3 \mathrm{D}$ block. More than 30 endpoints should be sampled in each sampling window (Rohrbaugh et al. 2002, Zeeb et al. 2013). For each network fracture realization, the amount of $\mathrm{N}_{0}$, $\mathrm{N}_{1}, \mathrm{~N}_{2}, \mathrm{~N}_{\text {total }}$ and visible trace length of each fracture in sampling window for $\mathrm{N}_{\mathrm{i}}$ is calculated. The maximum ratio of $\mathrm{N}_{2} / \mathrm{N}$ for joint sets no. 1, 2, 3 and 4 is 91 , 73, 63 and 60 percent in all realizations, respectively. In most cases, ratio of $\mathrm{N}_{2} / \mathrm{N}$ is smaller than 20 percent for all joint sets, too.

\section{PROCESSING OF SHAPE AND SIZE OF WINDOW AND SCANLINE EFFECT ON CHARACTERISTICS OF THE ROCK MASS}

\subsection{Mean trace length of fractures}

3.1.1 The equations of mean trace length of fracture The equations 1 to 9 (depending on the sampling method) in Table no. 1 are used to investigate the apparent and actual mean trace length of fracture. Each symbol in presented graphs is the average of five realization results. In simulations, the calculated mean trace of four joint sets by circular sampling is closed to the actual values except equations $7 \& 9$ and with square sampling, except for the equations $3 \& 4$. The error for each four joint sets is smaller than $15 \%$ in appropriate areal sampling windows. For instance, the mean trace length of joint set $\mathrm{J}_{2}$ with three sampling methods and all equations is shown in Figure 2. The true trace length $(1.1 \mathrm{~m})$ is shown by the dash line.

In maximum dimension of the sampling window area (areal sampling number 6), the maximum error $(15 \%)$ between the calculated value by equation 2 and the actual value is related to joint set $J_{3}$. This error is 


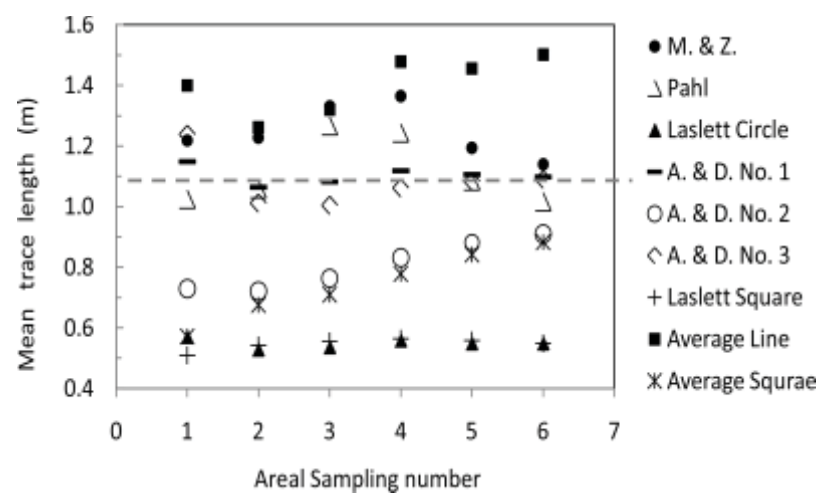

Figure 2. The apparent and actual mean trace length of $\mathrm{J}_{2}$ by scanline, square and circular sampling methods, simulation 1.

acceptable. So, the equations 2, 5, 6 and 8 are suitable for mean trace calculation and are not affected by the length and censoring biases. But, the equations 1, 3, 4, 7 and 9 aren't suitable for mean trace calculation. The Laslett's equation is appropriate for trace lengths that they obey negative exponential distribution (Priest 1993). For all joint sets the difference between equations 3 and 9 is small. Areal sampling method includes square and circular windows decreases the length and censoring bias almost equally in comparison with scanline.

In simulations and for all joint sets, the result of equation 7 is very different from the actual value. In equation 7 , by use of circular radius bigger than the actual trace length of $\mathrm{J}_{1}, \mathrm{~J}_{2}, \mathrm{~J}_{3}$ and $\mathrm{J}_{4}$ sets, the error is $23,34,42$ and $32 \%$ respectively. It's notable that, for joint sets $\mathrm{J}_{1}$ and $\mathrm{J}_{2}$ the calculated values get closer to the actual value by increasing of the sampling window size (the error is 8 and 18 percent, respectively). But, for other joint sets, the minimum size of the window should be 1.5 times more than the area sampling no. 6 . For all joint sets, the results of equation 7 are very close to the apparent mean trace length with square window and the maximum difference is equal to $7 \%$.

\subsubsection{Appropriate window sampling}

The value calculated by the equation 2 (square sampling) and the equations 5, 6 and 8 (circular sampling) are get close together for the fractures length smaller than 2 meter, But they depend on Fisher constant for longer fractures that will be explained in section 3-1-3.

The results of simulations show that for maximum $15 \%$ error, the area sampling nos. 1, 2, 3 to 4 and minimum no. 4 are appropriate for joint sets with trace length $0.5 \mathrm{~m}$, about $1 \mathrm{~m}$, about $2 \mathrm{~m}$ (depend on fisher constant) and bigger than $2 \mathrm{~m}$, respectively. For $\mathrm{J}_{4}$, the reason of the calculated trace length deviation $(10 \%$ to $15 \%$ error) in area sampling no. 7 is that the model and window sampling boundaries are close to each other (edge effect). This matter can be observed in Zhang's study that biggest sampling radius is $9 \mathrm{~m}$, dimension of model is $20 * 20 \mathrm{~m}$ and diameter of fracture is $2 \mathrm{~m}$ (Zhang et al. 2010). According to modeling, for fracture length longer than 2 meter it is recommend that for decreasing the error to $10 \%$, the ratio of model

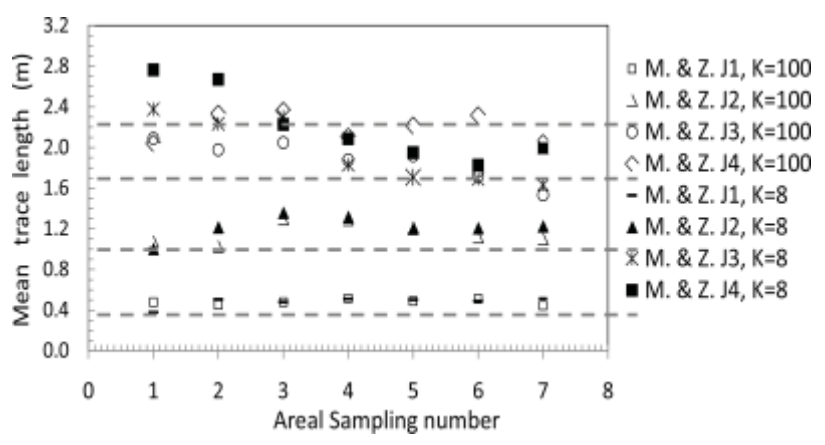

Figure 3. The mean trace length for all joint sets as regards the Fisher constant variation by the equation 5, the simulations $2 \& 3$.

dimension to circle radius and to dimension of square should be minimum 3.5 and 2 , respectively.

\subsubsection{Fisher constant effect on mean trace length}

As regards the comparison of the simulations 2 and 3 results, the variation of Fisher constant has not affected on the results of the equations 2 and 5 for small trace length $\left(J_{1}, J_{2}\right)$. But it has affected for the bigger trace length $\left(\mathrm{J}_{3}, \mathrm{~J}_{4}\right)$ in such a way that the results are $21 \%$ different for $\mathrm{k}=8$ and $\mathrm{k}=100$. The variation results of Fisher constant effect on the mean trace length of all joint sets are shown in Figure 3 by the equation 5 and the simulations $2 \& 3$. The difference between the equations $2 \& 5$ are maximum $8 \%$ and $21 \%$ for $\mathrm{k}=00$ and $\mathrm{k}=8$, respectively. If the Fisher constant for trace length bigger than 2 meter is smaller than 10 , the area sampling number should be minimum 5 for calculation of mean trace length. The theoretical mean trace length of join sets is shown by the dash line.

\subsection{Fracture trace density}

True density $\mathrm{P}_{20}$ is population density. In order to measure the true density, the numbers of fracture centers are necessary (Mauldon 1998). The number of fracture centers and then $\mathrm{P}_{20}$ can be calculated by 3DAVOSHT code. In this part, the equations 10 to 13 in Table 2 are considered to study apparent and actual density. In proper dimension of sampling window, the results of simulations show that the density calculated by square and circular windows, equations 11 and 13 are close to $\mathrm{P}_{20}$ and error is less than 4\%. For instance, the apparent and the true density calculated by square and circular windows for joint set $\mathrm{J}_{4}$ are shown in Figure 4. The true density $\left(\mathrm{P}_{20}\right)$ of joint sets is shown by the dash line.

Due to censoring effects for all joint sets, the apparent density is larger than the population density. Due to the fracture length, the apparent density calculated by the equations 10 and 12 are closer to $P_{20}$ with increases the sampling window dimensions, because of decrease in censoring.

In the maximum dimension of sampling number (6), the apparent density with square window for joint sets $\mathrm{J}_{1}, \mathrm{~J}_{2}, \mathrm{~J}_{3}$ and $\mathrm{J}_{4}$ are $10,16,27$ and $27 \%$ bigger than the true density. Meanwhile, the apparent density with 


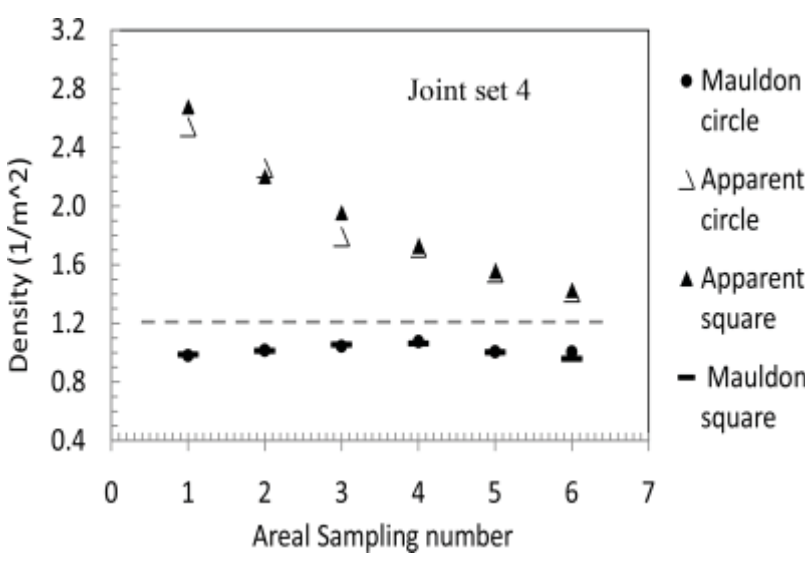

Figure 4. The apparent and true density calculated by square and circle window for the joint set $\mathrm{J}_{4}$, the simulation no. 1 .

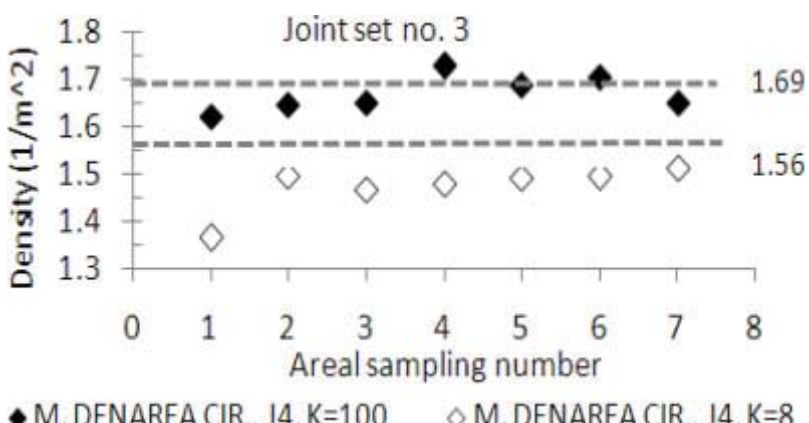

Figure 5. The density calculated by circular window sampling (equation 13) for joint set $J_{3}$ by variation of Fisher constant, the simulations 2 and 3.

circular window is somehow closer to the true density, comparing the square window. The apparent density is scale-dependent due to the edge effects (Mauldon 1999a, Brown 1981). While, in the estimator presented by Mauldon, the equations 11 and 13 are scale independent and automatically correct the censoring and length bias. So, for fractures length longer than $1 \mathrm{~m}$, assuming that the error bigger than $15 \%$ is not allowable, making use of the apparent density even in the sampling number 6 is not correct.

The result shows that in addition to the scale, the amount of Fisher constant effects on density, Figure 5. Taking into consideration between the simulation results of 2 and 3 , depending on the fracture length, the amount of $\mathrm{P}_{20}$ (by equation 13) in $\mathrm{K}=100$ is 5 to $10 \%$ bigger than the case $\mathrm{K}=8$. This matter is right for the equation no. 11, too. So, considering of the Fisher constant effect, the minimum areal sampling number for calculation the true density of fracture length of about $0.5 \mathrm{~m}$, about 1 to $2 \mathrm{~m}$ and about 2 to $3 \mathrm{~m}$ should be 2,3 or 4 and 5 respectively.

\subsection{Fracture trace length intensity}

True intensity $\left(\mathrm{P}_{21}\right)$ is population intensity. This part renders an account of the equations 14 to 18 in Table 3 related to the linear and areal intensity.

\subsubsection{Linear intensity of fracture trace}

Although scanline method calculates the linear intensity quickly, it is affected by the orientation, length, censoring bias and pattern heterogeneity (Rohrbaugh et al. 2002). The equations 14 and 15 are related to the linear intensity. According to the result of the simulation 1 , for the joint set $J_{1}$ with the dip larger than 80 degree, the estimated intensity is larger than $\mathrm{P}_{21}$. As regards the dip of joint sets $\mathrm{J}_{2}, \mathrm{~J}_{3}$ and $\mathrm{J}_{4}$ the scanline have less intersection with them; so, the value of $\mathrm{P}_{10}$ (equation 14) and the equation 15 have been estimated much less than $\mathrm{P}_{21}$. This is very obvious for $\mathrm{J}_{4}$. In the maximum dimension of sampling window by decreasing the joint set dip from 60 to 10 degree, the difference between $\mathrm{P}_{21}$ and $\mathrm{P}_{10}$ increase from $10 \%$ to $80 \%$. The reason of this matter is orientation bias. In view of the orientation bias, when the scanline or long axis of sampling window isn't perpendicular to the joint set the intensity is less estimated (Rohrbaugh et al. 2002).

\subsubsection{Areal intensity of fracture trace}

The equations 16 to 18 have been used for calculating the areal intensity. Since the areal intensity is two dimensional, it is more accurate than the linear one. Except for joint set $\mathrm{J}_{2}$, the result of the equations 16, 17 and 18 results are close together with a difference less than 5\%. This shows that the amount of apparent intensity, applying the circular and square window methods, is relatively equal to $\mathrm{P}_{21}$.

It is notable that, this difference is less than $20 \%$ for joint set $\mathrm{J}_{2}$ and decreases by increasing the fracture length. Areal intensity is independent from the sampling shape, but depends on the size of the area sampling. Assuming 15\% of error accepted, if the Fisher constant is 100 and the areal sampling number is 2 , the equation no. 18 is a suitable equation for all joint sets. When the areal sampling number is 3 , the error will be less than 7\%. In Pattern homogeneity, 15\% error is acceptable for true intensity (Rohrbaugh et al. 2002). According to the simulation 2 results, decreasing the Fisher constant to 8 will not have any effect on the results of joint sets $J_{1}, J_{2}$ and $J_{3}$ but in case of joint set $\mathrm{J}_{4}$, the areal sampling number 4 is necessary for the error less than $7 \%$.

Based on the results of the simulations 2 and 3 and the equation 18 , for all joint sets the variation of Fisher constant from 100 to 8 do not cause considerable variations in the intensity. The variation of $\mathrm{P}_{21}$ (true intensity) is $7 \%, 6 \%, 9 \%$ and $11 \%$ for joint sets $\mathrm{J}_{1}$, $\mathrm{J}_{2}, \mathrm{~J}_{3}$ and $\mathrm{J}_{4}$, respectively, Figure 6 . The true intensity $\left(\mathrm{P}_{21}\right)$ of joint set $\mathrm{J}_{2}$ is shown by the dash line. When the size and fracture intensity increase, efficiency of this estimator will be increased (Mauldon et al. 1999a, b).

If there is no equation between the density and trace length, the intensity can be considered as the product of the density and the mean length (Mauldon et al. 2001). When $\mathrm{K}=8$, the $\mathrm{P}_{21}$ is exactly the product of the true trace length and $\mathrm{P}_{20}$. Providing that, $\mathrm{K}$ increases to 100 , the difference of the above equality would be increase to $5 \%$ (ex. for $\mathrm{J}_{4}$ ). It seems, if the population intensity decreases, this difference would be increased. 


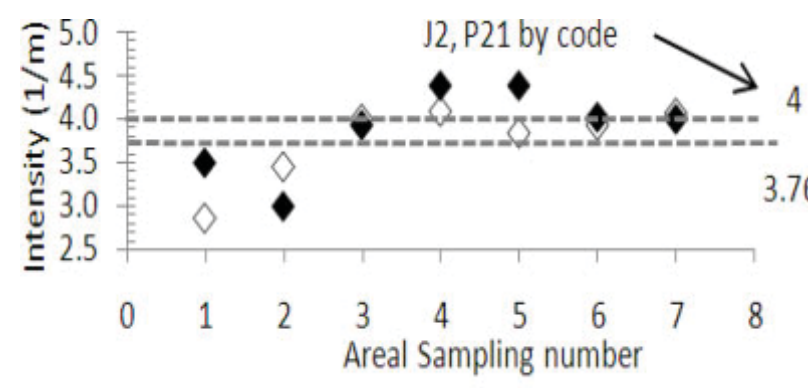

- Mauldon circle, $\mathrm{K}=100 \diamond$ Mauldon circle, $\mathrm{K}=8$

Figure 6. The areal intensity calculated by circular window sampling method (the equation 18) for joint set $\mathrm{J}_{2}$, the simulations 2 and 3.

\section{CONCLUSION}

Trace length, intensity and density are the most important characteristics of fractures that affects on the mechanical and hydraulic properties of the rock mass. The estimation of these parameters at site runs into some problems such as censoring and types of bias. These characteristics are estimated by three sampling methods.

The 3DDFNE code (3DAVOSHT) is developed to calculate the above characteristics. Considering the types of sampling method, this code can calculate the apparent and actual types of them based on various equations and estimators. The calculated trace length values based on equations and estimators of square sampling window (equation 2) and some estimators of circular window (equations 5, 6 and 8) for fractures trace length less than $2 \mathrm{~m}$ are close to each other by accepting the allowable error. But, the Fisher constant should be considered for fracture length longer than $2 \mathrm{~m}$. As regards the censoring effect, the amount of apparent density is larger than the population density. The results of circular and square sampling window equations (equations 11 and 13) are close to true density

In addition to the scale, the Fisher constant affects the density of traces. Depending on the fracture length when the Fisher constant is high, the amount of the real density is 5 to $10 \%$ higher than the real density with low Fisher constant. The apparent intensity calculated by circular and square window is nearly equal to the results of circular window estimator (equation 18). The optimum size of sampling window for calculating the trace length, intensity and density depends on length of joint and Fisher constant.

\section{REFERENCES}

Baecher, G.B., Lanney, N.S. \& Einstein, H.H. 1977. Statistical descriptions of rock properties and sampling. Proceedings of the U.S. symposium on rock mechanics 5C1.1-5c1.8.
Brown ET. Editor, 1981. ISRM suggested methods: rock characterization, testing and monitoring. London: Pergamon.

Dershowitz, S.D. \& Herda, H.H. 1992. Interpretation of fracture sacing and intensity. International Journal of Rock mechanics Tillerson \& Wawersik (eds), Balkema: Rotterdom.

Ferrero, A.M. \& Umili, G. 2011. Comparison of methods for estimating fracture size and intensity applied to Aiguille Marbee (Mont Blanc). International Journal of Rock Mechanics \& Mining Sciences 48: 1262-1270.

Kulatilake, P.H.S.W., Um J., Wang M., Escandon R.F. \& Narvaiz J. 2003. Stochastic fracture geometry modeling in 3-D including validations for a part of Arrowhead East Tunnel, California, USA. Engineering geology 70: 131-155.

Lei, Q., Latham, J.-P., Xiang, J., Tsang, C.-F., Lang, P. \& Guo, L. 2014. Effects of geomechanical changes on the validity of discrete fracture network representation of realistic twodimensional fractured rock. International Journal of Rock mechanics \& Mining sciences 70: 507-523.

Mauldon, M. 1998. Estimating mean fracture Trace length and density from observations in convex window. Int. J. Rock Mech. Rock Eng. 31 (4): 201-216.

Mauldon, M., Dunne, W.M. \& Rohrbaugh, Jr. M.B. 2001. Circular scanline and circular windows: new tools for characterizing the geometry of fracture traces. Journal of Structural Geology 23: 247-258.

Mauldon, M., Rohrbaugh, Jr. M.B., Dunne, W.M. \& Lawdermilk, W. 1999. Mean fracture trace length and density estimators using circular windows. Rock mechanics for industry, Balkema, Rotterdam, ISBN 9058090523.

Mauldon, M., Rohrbaugh, Jr. M.B., Dunne, W.M. \& Lawdermilk, W. 1999. Fracture intensity estimates using circular scanlines. Rock mechanics for industry, Balkema, Rotterdam, ISBN 9058090523.

Priest, S.D. 1993. Discontinuity analysis for rock engineering. London: Chapman and Hall.

Reeves, D.M., Parashar, R., Pohll, G., Carroll, R., Badger, T. \& Willoughby, K. 2013. The use of discrete facture network simulations in the design of horizontal hillslope drainage networks in fractured rock. Int. J. Engineering Geology 163: 132-143.

Rohrbaugh, Jr. M.B., Dunne, W.M. \& Mauldon, M. 2002. Estimating fracture trace intensity, density and mean length using circular scan lines and windows. The American Association of Petroleum Geologists. AAPG Bulletin 86 (12): 2089-2104.

Zeeb, C., Gomez-Rivas, E., Bons, P.D., Virgo, S. \& Blum, P. 2013. Fracture network evaluation program (FraNEP): A software for analyzing 2D fracture trace-line maps. Int. J. Computers \& Geosciences 60: 11-22.

Zhang, L. \& Ding, X. 2010. Variance of non-parametric rock fracture mean trace length estimator. International Journal of Rock Mechanics \& Mining Sciences 47: 1222-1228.

Zhang, L. \& Einstein, H.H. 2000. Estimating the intensity of rock discontinuities Int. J. Rock Mech. Min Sci., 37: 819-837.

Zheng, J., Deng, J., Zhang, G. \& Yang X. 2015. Validation of montcarlo simulation for discontinuity locations in space. Computers and Geotechnics 67: 103-109. 